\title{
Sister Society Meeting on Global Education Development and Collaboration:
}

\section{Meeting report}

Linus Chuang, Thomas Randall, Lynette Denny, Carolyn Johnston, Kathleen Schmeler, Allan

Covens, David Cibula, Michael Bookman, Sudhir Rawal, Denny DePetrillo, Joo-Hyun Nam, Annekathryn Goodman, Raj Naik, Ranjit Manchanda, David Gaffney, William Small, Carien Creutzberg, Carole Rattray, Vesna Kesic, René Paraja, Mary Eiken, Kristin Belleson, Robert Coleman, Richard Barakat, Edward Trimble, Michael Quinn

Globally, cervical cancer is the fourth most common cancer and the second leading cause of cancer death in women[1]. Approximately $85 \%$ of cervical cancers and related deaths are in low and middle income countries (LMIC) and although some advance has been made in cervical cancer prevention with screening and vaccination, it will be many years before an improvement in outcomes will be seen. With successful screening it can be expected that early stage diagnosis will be more common, allowing surgery to become a more important modality of treatment in addition to radiation and chemotherapy for locally advanced disease.

There are few cancer treatment training programs provided in LMICs and these are usually focused on urgent local needs. Such programs tend to be ad hoc and to be disadvantaged by lack of systematic cancer registry data to document improvement in outcomes, by lack of modern radiation equipment and of access to chemotherapeutic agents, and by suboptimal healthcare infrastructure including a lack of physicians[2, 3]. Despite these challenges, multiple groups and individuals have made significant efforts in establishing training programs. 
Some examples include Dr. Peter Heinz and his colleagues from the Dutch School of Gynecologic Oncology and Pelvic Surgery (http://www.dutchschool.nl) Netherlands, who developed an extensive program for fellows and gynecological oncology nurses in Indonesia, and the International Gynecologic Cancer Society (IGCS), which has awarded travel scholarships for younger faculty from low-resource settings to participate in the Dutch School programs. Dr. Barry Rosen and members of the Society of Gynecologic Oncology of Canada who have established training curricula on radical hysterectomy in Kenya and Mongolia and the Central American Gynecologic Oncology Education Program (CONEP) supported by IGCS and led by Dr. Kathleen Schmeler and her team from the MD Anderson Cancer Center (http://www.igcs.org/meetings/CONEP/CONEP.html) who have been teaching gynecologic oncology to residents in obstetrics and gynecology in Central America[4, 5]. In Bangladesh, a training program of surgical training, didactics, and development of infrastructure for cancer care of women has been developed by Dr Annekathryn Goodman and supported by Massachusetts General Hospital. Health Volunteers Overseas (HVO), which was established 30 years ago, has collaborated with the Society of Gynecological Oncology and the America Society of Clinical Oncology to send volunteers to Honduras, Vietnam, Paraguay, Nepal and Ghana (https://www.sgo.org/members-only/volunteer/health-volunteers-overseas/) [6].

A formal training program for regions where there are no gynecologic oncologists was formally established 3 years ago in Ghana by Dr. Carolyn Johnston and in Ethiopia jointly by Dr. Johnston and Dr. Ira Horowitz where the two years fellowship program in gynecologic oncology has already graduated its first four fellows. These formally trained fellows will serve as faculty to train more future fellows and to provide care for women with gynecologic cancers in Ethiopia. 
Given the lack of an overall strategy to meet the needs of LMICs, the leadership of the major gynecologic cancer societies and global health volunteers organized a meeting at the European Society of Gynecologic Oncology on October 23, 2015 aimed to identify common barriers to teaching and training and to identify synergies which would be useful in developing future programs.

Uniform primary challenges include language barriers, limited surgical equipment (especially for laparoscopy), inadequate internet access, lack of local support for sustainability (a common theme) in training programs, inadequate pathology and radiation oncology, and a global deficiency in identifying appropriate sites and personnel interested in partnering or developing training programs. Finance was, of course, a perennial problem. The teaching and career development of gynecologic oncologists in LMICs is hindered enormously by journals only being available in English, a barrier not only to learning but also to local publication.

Another significant theme was the lack of existing local faculty support and "buy in" of these volunteer programs, which often supplant existing teaching curricula, especially where local teaching staff are not actively involved. Inclusivity was seen as paramount to ensure the potential effect on the downgrading of the expertise of the local staff.

Many of the hospitals where the volunteers work are not equipped with a functioning internet service, precluding access to web based educational material and to tumor boards and other interactive and networking opportunities with the training partners.

Site selection has occasionally been challenging. Some hospitals have received more than one group of volunteers in the same specialty leading to a waste of the financial and intellectual investment by the volunteers. Most visitors pay for their own trips and spend in general 1-2 
weeks volunteering, often using their own vacation time. Other than a limited number of programs where there is philanthropic support, most receive some support from professional organizations supplemented by their local institutions and personal resources.

The way forward for building on successful programs and models of care firstly requires an awareness of what programs exist, how they are funded, what educational tools have been used, what has proven to be effective, and how success is measured. Careful planning including consultation with the local Ministry of Health, local educational governing bodies, and with the leaderships of key educational institutions is vital. Through these meetings, international professional societies are increasingly aware and committed to sharing programmatic initiatives and collaborating on meaningful solutions.

A sustainable gynecologic oncology training program depends on multiple facets including the support and the inclusion of the program into other local programs, a needs assessment prior to entry and exit placements of the trainees to provide appropriate resources where they are currently not available locally. The concept of developing regional centers of excellence is important as these can provide resource appropriate training whilst minimizing the "brain drain" of the trained specialists.

The development of a global curriculum which can be adapted with modification at different sites is urgently required. This template needs to be realistic, culturally sensitive and easily adaptable to local circumstances and needs. Although different countries have differing lengths of training requirements, have different approaches to surgery (eg, breast surgery included or only pelvic surgery undertaken), have differing access to radiation oncology and chemotherapy, and have variable expertise in radiology and pathology, it should be possible to establish a minimum training package adaptable to local situations and to be translated into the local language. 
Such a package will need to have the blessing of the major training providers and international organizations to carry any weight. Principles of cancer care including the importance of the tumor board and of palliative care can easily be included and emphasized.

In conclusion, education and training in gynecologic oncology will provide improved care for women with gynecologic cancers. To achieve this, the proper preparation of training sites and of trainers, the development of a global curriculum, the establishment of centers of excellence and the ability to measure outcomes will all be necessary. We should add educational programs to the NCI's Global Cancer Project Map (globalonc.org/Projects/global-cancer-projectmap/) where information is already collected relating to international cancer control and research efforts. A coordinated approach with the buy-in of leading gynecological cancer organizations will go some way to making a difference. This, together with the good will of so many volunteers, will make such organizations more relevant to the global community.

1. Ferlay, J., et al., Cancer incidence and mortality worldwide: sources, methods and major patterns in GLOBOCAN 2012. Int J Cancer, 2015. 136(5): p. E359-86.

2. del Carmen, M.G., L.W. Rice, and K.M. Schmeler, Global health perspective on gynecologic oncology. Gynecol Oncol, 2015. 137(2): p. 329-34.

3. Meara, J.G., et al., Global Surgery 2030: evidence and solutions for achieving health, welfare, and economic development. Int J Obstet Anesth, 2016. 25: p. 75-8.

4. Elit, L.M., et al., Teaching cervical cancer surgery in low- or middle-resource countries. Int J Gynecol Cancer, 2010. 20(9): p. 1604-8.

5. Schmeler, K.M., et al., The Central America Gynecologic Oncology Education Program (CONEP): improving gynecologic oncology education and training on a global scale. Gynecol Oncol, 2013. 129(3): p. 445-7.

6. Chuang, L., et al., Teaching gynecologic oncology in Low resource settings: a collaboration of health volunteers overseas and the society of gynecologic oncology. Gynecol Oncol, 2014. 135(3): p. 580-2. 
